\title{
AVALIAÇÃO DE DOIS SIMULADORES PARA PREDIÇÃO DA LIXIVIAÇÃO DE SULFONA DE ALDICARBE EM UM LATOSSOLO VERMELHO-AMARELO'
}

\author{
RÔMULO PENNA SCORZA JÚNIOR², RENÊLUÍS DE OLIVEIRARIGITANO³, \\ LUIZ ANTÔNIO LIMA ${ }^{4}$ e ANDERSON VITOR GOUVÊA ${ }^{5}$
}

\begin{abstract}
RESUMO - Este trabalho teve por objetivo o estudo da lixiviação de sulfona de aldicarbe em um Latossolo Vermelho-Amarelo, na região de Lavras, MG, para posterior comparação com os resultados obtidos pelos simuladores CALF e PESTLA. Os resultados mostraram alta lixiviação de sulfona de aldicarbe no solo em estudo. Aos 44 dias após a aplicação do inseticida e com uma lâmina de água acumulada de $241,4 \mathrm{~mm}$, a quantidade média de sulfona de aldicarbe remanescente nas colunas de solo de $45 \mathrm{~cm}$ de profundidade foi equivalente a 17,92\% $( \pm 5,88)$ da dose aplicada, enquanto os simuladores CALF e PESTLA estimaram 20,52\% e 37,82\%, respectivamente. O simulador CALF gerou melhores estimativas da quantidade média de sulfona de aldicarbe no perfil do solo nas diferentes datas de amostragem, enquanto o simulador PESTLA gerou melhores estimativas da distribuição do inseticida ao longo do perfil do solo, nas diferentes datas de amostragem.
\end{abstract}

Termos para indexação: simulação, aldicarbe, CALF, PESTLA, pesticidas no solo.

\section{EVALUATION OF TWO SIMULATORS TO PREDICT THE LEACHING OF ALDOXYCARB IN A RED-YELLOW LATOSSOL}

\begin{abstract}
This work was carried out to study the leaching of aldoxycarb in a Red Yellow Latossol in Lavras, Minas Gerais State, Brazil, and to compare the observed results with those obtained by the simulators CALF and PESTLA. The results showed a high leaching of aldoxycarb in the soil. After 44 days of the insecticide application and with $241.4 \mathrm{~mm}$ of accumulated water applied in each column, the remained quantity of aldoxycarb in the soil columns (45 cm long) was $17.92 \%( \pm 5.88)$ of the initial applied rate. However, the simulators CALF and PESTLA predicted $20.52 \%$ and $37.82 \%$, respectively. CALF simulator gave better predictions of the overall mean residue concentrations of aldoxycarb, and PESTLA simulator gave better prediction of residues distribution.
\end{abstract}

Index terms: simulation, aldicarb, CALF, PESTLA, pesticides in soil.

\section{INTRODUÇÃO}

Diante dos inúmeros casos de contaminação com resíduos de pesticidas em lençóis freáticos, poços e

\footnotetext{
${ }^{1}$ Aceito para publicação em 4 de março de 1999.

Extraído da Dissertação de Mestrado apresentada pelo primeiro autor à Universidade Federal de Lavras (UFLA).

${ }^{2}$ Eng. Agrôn., M.Sc., Environmental Sciences Department/WAU, Dreijenplein, 10, $6703 \mathrm{HB}$, Wageningen, The Netherlands. E-mail: romulo.scorza@bodhyg.benp.wau.nl

${ }^{3}$ Eng. Agrôn., Ph.D., Prof. Titular, Dep. de Entomologia, UFLA, Caixa Postal 37, CEP 37200-000 Lavras, MG E-mail: rigitano@ufla.br

${ }^{4}$ Eng. Agríc., Ph.D., Prof. Adjunto, Dep. de Engenharia, UFLA. E-mail: luizlima@ufla.br

${ }^{5}$ Eng. Agríc., Dep. de Entomologia, UFLA.
}

minas d'água (Jones \& Back, 1984; Jones et al., 1986, 1988; Domagalski \& Dubrovsky, 1992), pesquisadores no mundo inteiro têm somado esforços para estudar a lixiviação e degradação dos pesticidas em solos, permitindo elaborar questões sobre os riscos de contaminação ambiental. No entanto, o custo da condução de experimentos em campo para as diversas combinações de solo, clima e pesticidas torna-se bastante oneroso. Assim, simuladores como CALF (Nicholls et al., 1982b), PRZM/PRZM2 (Carsel et al., 1985; Mullins et al., 1993), LEACHM (Hutson \& Wagenet, 1992), PESTLA (Boesten \& Linden, 1991), CMLS (Nofziger \& Hornsby, 1986), GLEAMS (Leonard et al., 1987), MACRO (Jarvis, 1994), PELMO (Klein, 1995), PESTFADE (Clemente et al., 1993) e RZWQM (Ahuja et al., 1996) 
foram desenvolvidos, permitindo, através da utilização de um microcomputador, simular a lixiviação de pesticidas no solo, para possibilitar a previsão de contaminação ambiental.

Trabalhos mostrando a avaliação de alguns desses simuladores para predição da lixiviação de pesticidas em solos foram realizados principalmente na América do Norte e Europa (Carsel et al., 1986; Pennell et al., 1990; Loague \& Green, 1991; Parrish et al., 1992; Shirmohammadi \& Knisel, 1994; Walker et al., 1996). Países como a Holanda (Brouwer, 1994) e Alemanha (Klein, 1995) têm utilizado alguns desses simuladores como instrumento para amparar os órgãos competentes com relação ao impacto ambiental e registro de pesticidas.

Sulfona de aldicarbe é um produto da transformação do aldicarbe, o qual é muito utilizado em várias culturas de importância econômica, como café, citros, batata e algodão.

Este trabalho teve como objetivo estudar a lixiviação de sulfona de aldicarbe em um Latossolo Vermelho-Amarelo na região de Lavras, MG, utilizando colunas de solo não desestruturado, no campo, para posterior comparação com os resultados obtidos pelos simuladores CALF e PESTLA.

\section{MATERIAL E MÉTODOS}

O experimento foi instalado no dia 16/4/1996 em uma cultura de café da cultivar Icatú Vermelho MG 2942, com idade de, aproximadamente, quatro anos, pertencente à Empresa de Pesquisa Agropecuária de Minas Gerais (EPAMIG) em Lavras, MG. A área experimental consistiu de 4,00 x 7,10 m, sendo o solo do tipo Latossolo Vermelho-Amarelo, o qual foi gradeado no mês de agosto de 1995, para controle das ervas daninhas. A Tabela 1 mostra as porcentagens de areia, silte, argila e matéria orgânica nas diferentes profundidades do solo estudado.

Dezoito tubos de aço inoxidável $(50 \mathrm{~cm}$ de comprimento, $9 \mathrm{~cm}$ de diâmetro interno e $2 \mathrm{~mm}$ de espessura) foram introduzidos nas entrelinhas da cultura de café com auxílio de uma marreta, deixando-se $5 \mathrm{~cm}$ de sua parte superior acima da superfície do solo. A distância entre os tubos foi de $50 \mathrm{~cm}$, e estes, distribuídos em duas entrelinhas. No dia 3/6/1996, foram aplicados, no centro de cada uma das colunas, $100 \mathrm{mg}$ de sulfona de aldicarbe, diluídas em $5 \mathrm{~mL}$ de acetona. Em cada data de amostragem (7, 15, 22, 30, 37 e 44 dias após a aplicação de sulfona de aldicarbe), três colunas de solo não desestruturado foram retiradas com auxílio de uma cavadeira manual, as quais foram levadas ao laboratório para retirada de amostras de solo em intervalos de $5 \mathrm{~cm}$. Essas amostras de solo foram colocadas em sacos de plástico rotulados com a identificação, homogeneizadas e armazenadas em um congelador a uma temperatura de aproximadamente $-15^{\circ} \mathrm{C}$.

O método de extração de sulfona de aldicarbe das amostras de solo foi baseado no trabalho desenvolvido por Nicholls et al. (1982a). Cem gramas da amostra de solo foram transferidos para um erlenmeyer de $500 \mathrm{~mL}$, em seguida adicionando-se-lhes $200 \mathrm{~mL}$ de acetona. As amostras foram submetidas à agitação (150 rpm) por uma hora, e em seguida, deixadas em repouso, para separação das fases, por um período de uma hora. Do sobrenadante, retirou-se uma alíquota de $40 \mathrm{~mL}$, sendo a acetona evaporada em um rotavapor. A fase líquida remanescente foi transferida para um tubo de centrífuga com tampa, cujo volume foi completado com água destilada para 7,5 mL. Em seguida, realizou-se a partição líquido-líquido, agitando o tubo de centrífuga por um minuto, com três frações sucessivas de 7,5 mL de acetato de etila. Finalmente, o acetato de etila foi evaporado, e os resíduos de sulfona de aldicarbe foram transferidos em solução cetônica para um tubo graduado, com o volume final completado para $4 \mathrm{~mL}$.

Para determinação de sulfona de aldicarbe, foram injetados $2 \mu \mathrm{L}$ da amostra em um cromatógrafo a gás, modelo CG 370, equipado com um detector fotométrico de chama, operando com filtro de $394 \mathrm{~nm}$. Utilizou-se uma coluna de vidro SP 1000 (5\%) em Supelcoport 100/120. Os fluxos do gás de arraste (nitrogênio) e dos gases auxiliares, hidrogênio e ar sintético, foram de $50 \mathrm{~mL} / \mathrm{min}$, $171 \mathrm{~mL} / \mathrm{min}$, e $240 \mathrm{~mL} / \mathrm{min}$, respectivamente. As temperaturas da coluna, do vaporizador e do detector, foram $228^{\circ} \mathrm{C}, 232^{\circ} \mathrm{C}$ e $242^{\circ} \mathrm{C}$, respectivamente. A determinação de sulfona de aldicarbe foi realizada mediante a comparação das alturas dos picos nas amostras com a curva de calibração, a qual foi obtida injetando-se $2 \mu \mathrm{L}$ das soluções-padrão que continham concentrações variando de 2,5 a $20 \mathrm{ng} / \mu \mathrm{L}$. O padrão analítico foi obtido junto à Agência de Proteção Ambiental dos Estados Unidos (EPA) com pureza acima de $99 \%$.

O grau de umidade das amostras de solo foi determinado por gravimetria. De cada amostra foram retirados $50 \mathrm{~g}$ de solo, que foram colocados em um becker previamente tarado, e levados para a estufa, onde permaneceram por 24 horas, a uma temperatura de $105^{\circ} \mathrm{C}$.

Como a instalação do experimento se deu em uma época de pouca ou quase nenhuma chuva, foram realizadas simulações de chuva, com recipientes de plástico cilíndricos contendo furos na sua parte inferior, para saída de 
pequenas gotas de água. Esses recipientes de plástico foram encaixados na parte superior das colunas. Na superfície do solo, em cada coluna, colocou-se um pedaço circular (7 cm de diâmetro) de fibra sintética (Bidim), para garantir uma distribuição homogênea da água. No dia 4/6/96, foram aplicados $50 \mathrm{~mm}$ de água em cada coluna de solo, e nos dias 12/6/96, 19/6/96, 26/6/96, 3/7/96, 5/7/96, 10/7/96 e 12/7/96, apenas $25 \mathrm{~mm}$. Durante o período experimental, ocorreu apenas uma precipitação (28/6/96), com total de $16,4 \mathrm{~mm}$ medidos por um pluviômetro instalado no interior da parcela experimental.

Os dois simuladores utilizados neste trabalho diferemse principalmente com relação à abordagem matemática do fluxo da água no solo e do transporte de pesticidas. Para descrever o fluxo da água no solo, o simulador CALF utiliza o conceito de capacitância, enquanto o simulador PESTLA utiliza a equação de Richards. Já com relação ao transporte de pesticidas, o simulador CALF considera apenas o transporte convectivo, enquanto o simulador PESTLA utiliza a equação de convecção-dispersão.

Os valores dos parâmetros necessários para utilização dos simuladores CALF e PESTLA nas condições de solo e clima estudadas são mostrados nas Tabelas 2 e 3, respectivamente.

Para avaliação da qualidade dos ajustes dos valores obtidos pelos simuladores CALF e PESTLA aos valores observados, utilizou-se, além da análise gráfica, a análise de regressão linear e três índices propostos por Walker et al. (1996), ou seja:

Coeficiente de massa residual (CMR): $\sum\left(\mathrm{P}_{\mathrm{i}}-\mathrm{O}_{\mathrm{i}}\right) / \sum \mathrm{O}_{\mathrm{i}}$, onde $\mathrm{P}_{\mathrm{i}}$ corresponde ao valor da concentração do pesticida na profundidade $\mathrm{i}$, obtido pelo simulador, e $\mathrm{O}_{\mathrm{i}}$ corresponde ao valor observado da concentração do pesticida na profundidade i. Este índice compara os níveis de resíduos, sem levar em consideração a sua distribuição no solo. Um valor de CMR próximo de zero significa uma boa estimativa da quantidade média do pesticida remanescente no perfil do solo;

Coeficiente de forma $(\mathrm{CF}): \sum\left(\mathrm{O}_{\mathrm{i}}-\overline{\mathrm{O}}\right)^{2} / \Sigma\left(\mathrm{P}_{\mathrm{i}}-\overline{\mathrm{P}}\right)^{2}$,

onde $\overline{\mathrm{O}}$ e $\overline{\mathrm{P}}$ representam as médias dos valores observados e dos obtidos por simulação da concentração do pesticida, respectivamente. Valores de CF próximos a 1,0 significam semelhança na forma das curvas, mas não necessariamente uma semelhança com relação à posição no perfil do solo;

Eficiência da simulação (ES): $1-\sum\left(\mathrm{O}_{\mathrm{i}}-\mathrm{P}_{\mathrm{i}}\right)^{2} / \Sigma\left(\mathrm{O}_{\mathrm{i}}-\overline{\mathrm{O}}\right)^{2}$.

Este índice exprime o ajuste total entre os valores simulados e os observados. Valores de ES próximos a 1,0 significam ajuste perfeito entre os dados observados e preditos da concentração do pesticida nas diferentes profundidades do solo, ao passo que valores menores que zero mostram predição inadequada, sendo preferível utilizar como estimativa a média dos valores observados.

Com relação à análise de regressão linear entre os valores preditos e observados da concentração do pesticida nas diferentes profundidades, espera-se, para um perfeito ajuste entre esses valores, um coeficiente angular igual a 1,0 , uma interseção igual a zero, e coeficiente de determinação $\left(\mathrm{r}^{2}\right)$ igual a 1,0 .

\section{RESULTADOS E DISCUSSÃO}

A Fig. 1 mostra os valores observados, e os valores obtidos pelos simuladores CALF e PESTLA da lixiviação de sulfona de aldicarbe no solo estudado. Aos sete dias após a aplicação de sulfona de aldicarbe

TABELA 1. Porcentagem de areia, silte, argila e matéria orgânica (M.O.) nas diferentes profundidades do solo em estudo.

\begin{tabular}{ccccc}
\hline Profundidade $(\mathrm{cm})$ & Areia $(\%)$ & Silte $(\%)$ & Argila (\%) & M.O. $(\%)$ \\
\hline $0-5$ & 46,00 & 17,00 & 37,00 & 3,10 \\
$5-10$ & 44,00 & 15,00 & 41,00 & 2,50 \\
$10-15$ & 44,00 & 13,00 & 43,00 & 2,10 \\
$15-20$ & 44,00 & 13,00 & 43,00 & 2,00 \\
$20-25$ & 42,00 & 15,00 & 43,00 & 1,80 \\
$25-30$ & 44,00 & 13,00 & 43,00 & 1,60 \\
$30-35$ & 42,00 & 15,00 & 43,00 & 1,50 \\
$35-40$ & 44,00 & 11,00 & 45,00 & 1,40 \\
$40-45$ & 42,00 & 13,00 & 45,00 & 1,40 \\
\hline Média & $43,55 \pm 1,33$ & $13,88 \pm 1,76$ & $42,55 \pm 2,40$ & $1,93 \pm 0,57$ \\
\hline
\end{tabular}


TABELA 2. Valores de parâmetros utilizados pelo simulador CALF (Nicholls et al., 1982b) para simulação da lixiviação de sulfona de aldicarbe nas condições de solo e clima estudadas.

\begin{tabular}{lr}
\hline Característica & Valor \\
\hline Umidade na capacidade de campo (\% de peso) & 21,65 \\
Umidade do solo a 2 atm (\% de peso) & 12,58 \\
Umidade inicial do solo (\% de peso) & 17,39 \\
$\mathrm{~K}_{\mathrm{d}}(\mathrm{mL} / \mathrm{g})$ & $0,04^{1}$ \\
Solubilidade em água $(\mathrm{mg} / \mathrm{L})$ & $9.000,00^{2}$ \\
Densidade do solo $\left(\mathrm{g} / \mathrm{cm}^{3}\right)$ & 1,24 \\
Altitude (m) & 910,00 \\
Latitude (graus) & 21,00 \\
Meia-vida (dias) & $51,00^{3}$ \\
Constante A & $1.104,00^{4}$ \\
Constante B & $-0,85^{4}$ \\
Energia de ativação de sulfona de aldicarbe $(\mathrm{cal} / \mathrm{mol})$ & $17.157,00^{5}$ \\
Temperatura média do solo $\left({ }^{\circ} \mathrm{C}\right)$ & 18,60 \\
\hline
\end{tabular}

1 Estimado com base na equação de Goring (1962): $\mathrm{K}_{\mathrm{d}}=\mathrm{K}_{\mathrm{om}} \times \mathrm{MO}(\%) / 100$.

2 Worthing (1983).

3 Estimado com base na quantidade remanescente total de sulfona de aldicarbe aos 7,15 e 22 dias.

${ }^{4}$ Descrevem a influência da umidade na degradação (Nicholls et al., 1982a).

5 Nicholls et al. (1982a).

TABELA 3. Valores de parâmetros utilizados pelo simulador PESTLA (Boesten \& Linden, 1991) para simulação da lixiviação de sulfona de aldicarbe nas condições de solo e clima estudadas.

\begin{tabular}{lr}
\hline Característica & Valor \\
\hline Coeficiente de difusão do pesticida na água $\left(\mathrm{cm}^{2} / \mathrm{dia}\right)$ & $0,40^{1}$ \\
Dispersividade $(\mathrm{cm})$ & $5,34^{2}$ \\
$\mathrm{~K}_{\text {om }}\left(\mathrm{dm}^{3} / \mathrm{kg}\right)$ & $1,25^{3}$ \\
Expoente N da equação de Freundlich & $1,00^{4}$ \\
Meia-vida de referência (dias) & $38,00^{5}$ \\
$\mathrm{~B}$ & $0,70^{6}$ \\
$\gamma\left(\mathrm{K}^{-1}\right)$ & $0,08^{7}$ \\
Umidade de saturação $\left(\mathrm{cm}^{3} / \mathrm{cm}^{3}\right)$ & 0,53 \\
Umidade residual $\left(\mathrm{cm}^{3} / \mathrm{cm}^{3}\right)$ & 0,10 \\
Condutividade hidráulica saturada (cm/dia) & $400,80^{8}$ \\
$\mathrm{~L}$ & $0,50^{9}$ \\
$\alpha$ & $0,32^{9}$ \\
$\mathrm{~N}$ & $1,37^{9}$ \\
\hline 1 Boesten (1993). & \\
2 Corrêa (1996). & \\
3 Estimado com base na equação de Briggs (1981): log $\mathrm{K}_{\text {om }}=0,52 \times$ log $\mathrm{K}_{\text {ow }}+0,62$. & \\
4 Fixado para garantir a semelhança entre os dois simuladores quanto à abordagem matemática do processo de adsorção. \\
5 Valor da meia-vida considerando umidade do solo a 0,1 atm e temperatura igual a 20 ${ }^{\circ} \mathrm{C}$; estimado com base nos trabalhos de Piffer \& Rigitano (1991) \\
e Barbosa \& Rigitano (1994). \\
6 Parâmetro do modelo matemático utilizado para descrever a influência da umidade do solo na taxa de transformação (Boesten, 1993). \\
7 Parâmetro do modelo matemático utilizado para descrever a influência da temperatura do solo na taxa de transformação (Boesten, 1993). \\
8 Lima (1987). \\
9 Parâmetros obtidos através do ajuste dos dados de retenção de água do solo estudado à equação de Genuchten (1980).
\end{tabular}



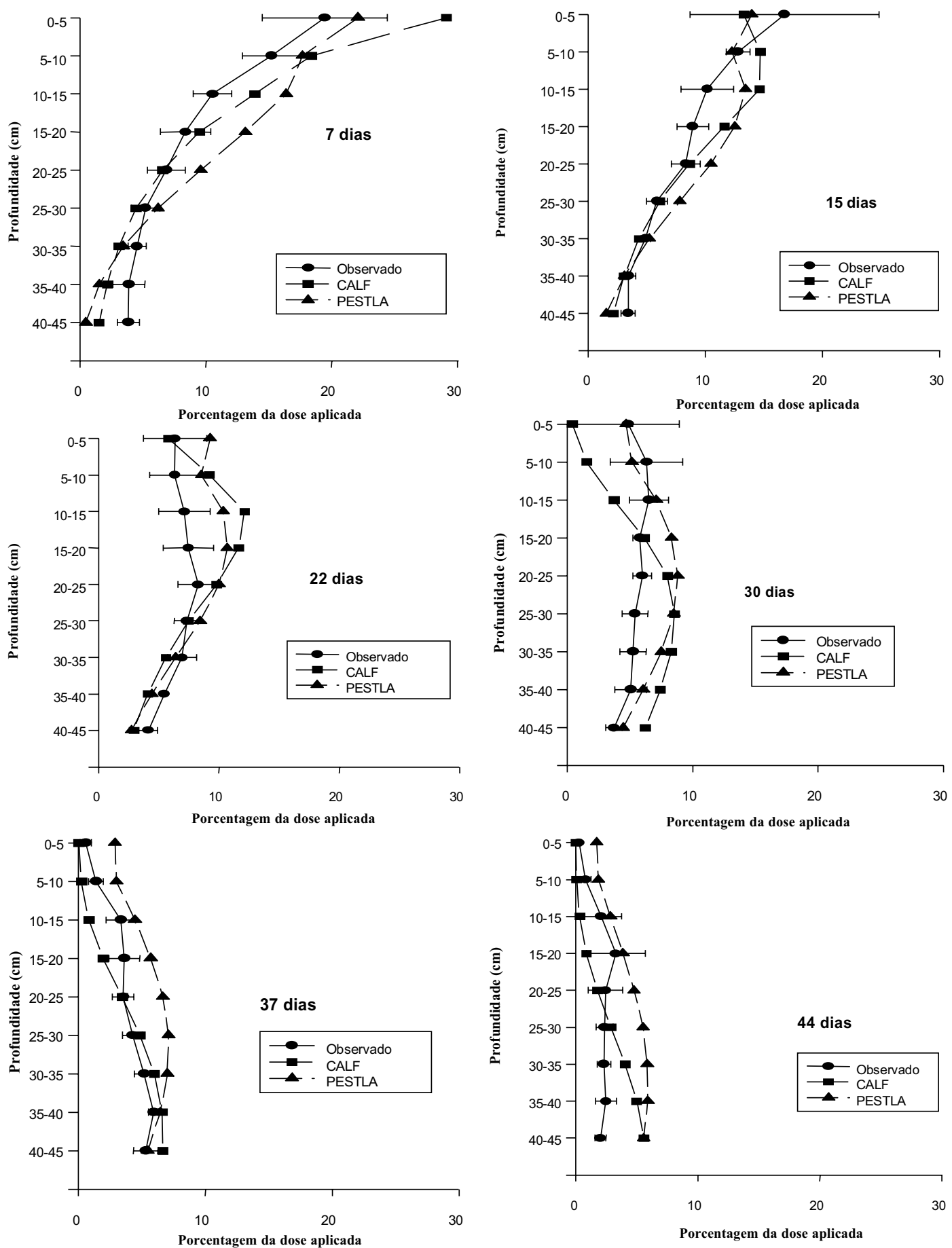

FIG. 1. Valores observados e preditos da lixiviação de sulfona de aldicarbe pelos simuladores CALF (Nicholls et al., 1982b) e PESTLA (Boesten \& Linden, 1991) em um Latossolo Vermelho-Amarelo após 7, 15, 22, 30, 37 e 44 dias da aplicação do composto (barras de erro representam o desvio-padrão dos dados). 
e com lâmina de água aplicada de $50 \mathrm{~mm}$, observouse intensa lixiviação do composto, embora a maior parte deste ainda se concentrasse nos primeiros $5 \mathrm{~cm}$ do solo. Esta alta lixiviação inicial de sulfona de aldicarbe se deve tanto ao baixo coeficiente de adsorção do composto, em função de sua alta polaridade (Briggs, 1981), como também à estrutura característica do solo em estudo, que resulta em maior número de macroporos, e, conseqüentemente, maior permeabilidade. Oliveira et al. (1992) citam que o Latossolo Vermelho-Amarelo se caracteriza por ser um solo poroso com alta taxa de infiltração.

Já aos 22 dias, observou-se maior concentração do composto entre os $20-30 \mathrm{~cm}$ de profundidade, sendo que a significativa diminuição deste, nos primeiros $15 \mathrm{~cm}$, é atribuída à lixiviação e à degradação mais intensa na superfície, como conseqüência de maior atividade microbiana e maiores temperaturas (Lightfoot et al., 1987).

$\mathrm{Na}$ última data de amostragem (44 dias), observaram-se quantidades pequenas (menores que 3,5\% da dose aplicada) de sulfona de aldicarbe nas diferentes profundidades. Esta pequena quantidade do composto encontrada deve-se ao fato de que grande parte foi lixiviada para profundidades superiores a $45 \mathrm{~cm}$, além da degradação do produto.

Os resultados apresentados neste trabalho com relação à lixiviação de sulfona de aldicarbe diferem dos encontrados por Piffer \& Rigitano (1991), que estudaram a lixiviação desse composto em um Latossolo Roxo e em um Podzólico Vermelho-Amarelo na região de Lavras, MG. Estes autores observaram maiores concentrações de sulfona de aldicarbe nos primeiros $5 \mathrm{~cm}$ de profundidade até o $42^{\circ}$ dia após a aplicação do composto, o que não foi observado no presente estudo. Essas diferenças são atribuídas à retenção de sulfona de aldicarbe no interior dos grânulos do produto comercial utilizado por Piffer \& Rigitano (1991), ao passo que no presente estudo o composto foi aplicado dissolvido em acetona, ficando, assim, prontamente disponível para ser lixiviado. É importante ressaltar que a lâmina de água aplicada nos dois estudos foi semelhante até o $42^{\circ}$ dia.

Na Fig. 1 observa-se que ambos os simuladores testados apresentam algumas discrepâncias entre os valores observados e os obtidos por simulação. Aos sete dias, verifica-se que o simulador CALF superestimou a concentração de sulfona de aldicarbe no intervalo de $0-20 \mathrm{~cm}$ e subestimou-a no intervalo de $25-45 \mathrm{~cm}$. Já o simulador PESTLA superestimou a concentração do composto no intervalo de $0-30 \mathrm{~cm} \mathrm{e}$ subestimou-a no intervalo de $35-45 \mathrm{~cm}$. Ambos os simuladores subestimaram a lixiviação de sulfona de aldicarbe, já que a quantidade transportada além dos $45 \mathrm{~cm}$ foi superior às obtidas pelos modelos. Esta subestimativa da lixiviação pode ser explicada pelo fato de os simuladores testados considerarem o processo de adsorção como um equilíbrio instantâneo entre a concentração do pesticida na fase aquosa e na fase sólida do solo. No entanto, a estrutura característica do Latossolo Vermelho-Amarelo favorece uma alta velocidade da água através dos macroporos, não permitindo, assim, que haja tempo suficiente para que ocorra o equilíbrio da concentração do pesticida na fase aquosa e sólida do solo (Rao \& Jessup, 1983). Bilkert \& Rao (1985) observaram que valores de $\mathrm{K}_{\mathrm{d}}$ (coeficiente de distribuição do pesticida entre os sólidos e a solução do solo), menores que os obtidos em laboratório pelos métodos de agitação das fases do solo, foram necessários para uma melhor predição da lixiviação de aldicarbe em condições de campo.

Aos 22 dias, o simulador CALF estimou maior concentração do composto na faixa de $5-25 \mathrm{~cm}$, enquanto o simulador PESTLA estimou maior concentração na faixa de $0-30 \mathrm{~cm}$. No entanto, os valores observados mostraram maior concentração de sulfona de aldicarbe na faixa de $20-30 \mathrm{~cm}$. Já no 370 dia, observa-se que o simulador PESTLA superestimou a concentração de sulfona de aldicarbe na faixa de $0-35 \mathrm{~cm}$, enquanto o simulador CALF apresentou tendência a superestimar a lixiviação do produto abaixo dos $30 \mathrm{~cm}$ de profundidade. No intervalo de 5-20 cm, o simulador CALF subestimou a concentração do composto.

O simulador PESTLA superestimou a concentração de sulfona de aldicarbe ao longo de todo o perfil do solo na última data de amostragem (44 dias), ao passo que o simulador CALF subestimou a concentração do composto no intervalo de 5-20 cm e superestimou-a no intervalo de $30-45 \mathrm{~cm}$ (Fig. 1).

As estimativas dos coeficientes de regressão (a), das interseções (b) e dos coeficientes de deter- 
minação $\left(\mathrm{r}^{2}\right)$, obtidos através da análise de regressão linear entre os dados preditos e os dados observados, são mostrados na Tabela 4. As duas regressões foram significativas $(\mathrm{P}<0,05)$. Observa-se que os valores de $\mathrm{r}^{2}$ foram bastante próximos, indicando assim uma similaridade na qualidade do ajuste entre os valores observados e os obtidos por ambos os simuladores testados. Walker et al. (1996), testando a nova versão do simulador CALF, renomeado para VARLEACH (Walker \& Hollis, 1994), na simulação da lixiviação de três herbicidas, observaram valores de $\mathrm{r}^{2}$ variando de $29,2 \%$ a $84,2 \%$.

A Tabela 5 mostra os valores do coeficiente de massa residual (CMR), do coeficiente de forma (CF) e da eficiência da simulação (ES) derivados da avaliação da qualidade do ajuste dos dados obtidos pelos simuladores CALF e PESTLA durante todo o período experimental. Observa-se grande variabilidade entre os simuladores testados e datas de amostragem com relação ao ajuste entre os valores simulados e os valores observados.
Quanto ao simulador PESTLA, verifica-se que com o decorrer do tempo, os valores de CMR se afastaram de zero, indicando, assim, tendência a estimativas menos confiáveis dos níveis de resíduos médios de sulfona de aldicarbe no perfil do solo. Com relação ao simulador CALF, os valores de CMR em todas as datas de amostragem foram inferiores aos valores do simulador PESTLA, o que confirma a capacidade deste simulador em gerar estimativas mais confiáveis da quantidade remanescente média de sulfona de aldicarbe no perfil do solo estudado. Estes resultados são semelhantes aos observados por Walker et al. (1996), que verificaram que o simulador VARLEACH mostrou-se mais confiável, em comparação com os simuladores PRZM2 e LEACHM, em vista da predição da quantidade remanescente média dos herbicidas alachlor, atrazina e metribuzin em um solo da Inglaterra.

Os valores de CF mostram que o simulador PESTLA, em comparação com o simulador CALF, gerou formas das curvas da distribuição de sulfona

TABELA 4. Estimativas dos coeficientes angulares (a), das interseções (b) e dos coeficientes de determinação $\left(r^{2}\right)$, obtidos através da regressão linear entre os dados preditos e observados da lixiviação de sulfona de aldicarbe.

\begin{tabular}{lcrrr}
\hline Modelo & $\mathrm{n}^{1}$ & $\mathrm{a}$ & $\mathrm{b}$ & $\mathrm{r}^{2}$ \\
\hline CALF & 54 & $1,2594 \pm 0,2006^{2}$ & $-1,2340 \pm 1,3845$ & $75,33 \%$ \\
PESTLA & 54 & $1,001 \pm 0,1529$ & $1,5286 \pm 1,0531$ & $76,95 \%$ \\
\hline
\end{tabular}

${ }^{1}$ Número de observações

${ }^{2}$ Intervalo de confiança a $95 \%$.

TABELA 5. Valores do coeficiente de massa residual (CMR), do coeficiente de forma (CF) e da eficiência da simulação (ES) para os simuladores CALF e PESTLA em todas as datas de amostragem.

\begin{tabular}{|c|c|c|c|c|c|c|}
\hline \multirow[t]{2}{*}{ Dias } & \multicolumn{3}{|c|}{ CALF } & \multicolumn{3}{|c|}{ PESTLA } \\
\hline & CMR & $\mathrm{CF}$ & ES & CMR & $\mathrm{CF}$ & ES \\
\hline 7 & 0,139 & 0,358 & 0,474 & 0,163 & 0,506 & 0,599 \\
\hline 15 & 0,053 & 0,794 & 0,718 & 0,078 & 0,911 & 0,727 \\
\hline 22 & 0,156 & 0,140 & $-3,887$ & 0,192 & 0,193 & $-2,497$ \\
\hline 30 & 0,028 & 0,080 & $-20,110$ & 0,240 & 0,255 & $-4,579$ \\
\hline 37 & $-0,077$ & 0,424 & 0,447 & 0,473 & 1,188 & $-0,389$ \\
\hline 44 & 0,145 & 0,177 & $-3,832$ & 1,110 & 0,287 & $-7,499$ \\
\hline Global & 0,020 & 0,479 & 0,475 & 0,247 & 0,760 & 0,597 \\
\hline
\end{tabular}


de aldicarbe ao longo do perfil do solo mais próximas do observado no campo, o que não indica necessariamente a mesma posição no perfil do solo.

Com relação aos valores de ES, verifica-se que houve aumento da eficiência da simulação em ambos os simuladores quando se comparam os valores obtidos aos sete e 15 dias. Isto pode ser observado também na Fig. 1. Outro ponto importante refere-se à similaridade dos dois simuladores, com relação à eficiência da simulação nos 15 dias. Os valores de ES, em todas as datas de amostragem, confirmaram a capacidade do simulador PESTLA em gerar estimativas mais confiáveis da lixiviação de sulfona de aldicarbe nas condições estudadas, conforme mostrado através dos valores de $\mathrm{r}^{2}$. No entanto, os valores negativos de ES, nas últimas datas de amostragem, com exceção dos 37 dias relativos ao simulador CALF, mostram que as estimativas geradas pelos simuladores nestas datas são menos confiáveis do que simplesmente a média dos valores observados.

É importante ressaltar que as diferenças verificadas na qualidade do ajuste entre os valores obtidos pelos simuladores e observados no campo têm como uma das causas a diferença na abordagem matemática, em cada simulador, dos processos que descrevem a lixiviação de pesticidas no solo. No entanto, a identificação e quantificação da influência de cada processo separadamente estava além do objetivo deste trabalho, já que dados mais detalhados seriam necessários.

\section{CONCLUSÕES}

1. O inseticida-nematicida sulfona de aldicarbe confirma ser um composto com um alto potencial de lixiviação no solo estudado.

2. O simulador CALF gera melhores estimativas da quantidade média de sulfona de aldicarbe no perfil do solo, nas diferentes datas de amostragem, quando comparado ao simulador PESTLA.

3. O simulador PESTLA gera melhores estimativas da distribuição de sulfona de aldicarbe ao longo do perfil do solo em todas as datas de amostragem quando comparado ao simulador CALF.

4. De maneira geral, o simulador PESTLA é indicado para predição da lixiviação de sulfona de aldicarbe nas condições de solo e clima estudadas.

\section{REFERÊNCIAS}

AHUJA, L.R.; MA, L.Q.; ROJAS, K.W.; BOESTEN, J.J.T.I.; FARAHANI, H.J. A field test of root zone water quality model - pesticide and bromide behavior. Pesticide Science, Oxford, v.48, n.2, p.101-108, Oct. 1996.

BARBOSA, T.M.L.; RIGITANO, R.L.O. Influência da classe e profundidade do solo na degradação do inseticida-nematicida aldicarbe. Pesquisa Agropecuária Brasileira, Brasília, v.29, n.6, p.955-960, jun. 1994.

BILKERT, J.N.; RAO, P.S.C. Sorption and leaching of three nonfumigant nematicides in soils. Journal of Environmental Science and Health, New York, v.20-B, n.1, p.1-26, 1985.

BOESTEN, J.J.T.I. Users manual for version 2.3 of PESTLA. Wageningen : SC-DLO, 1993. 39p.

BOESTEN, J.J.T.I.; LINDEN, A.M.A.van der. Modeling the influence of sorption and transformation on pesticide leaching and persistence. Journal of Environmental Quality, Madison, v.20, p.425-435, 1991.

BRIGGS, G.G. Theoretical and experimental relationships between soil, adsorption, octanol-water partition coefficients, water solubilities, bioconcentration factors, and the parachor. Journal of Agricultural and Food Chemistry, Washington, v.29, n.5, p.10501059, Sept./Oct. 1981.

BROUWER, W.W.M. Use of simulation models for registration purposes: evaluation of pesticide leaching to groundwater in the Netherlands. Journal of Environmental Science and Health, New York, v.29A, p.1117-1132, 1994.

CARSEL, R.F.; MULKEY, L.A.; LORBER, M.N.; BASKIN, L.B. The Pesticide Root Zone Model (PRZM): a procedure for evaluating pesticide leaching threats to groundwater. Ecological Modelling, Amsterdam, v.30, p.46-69, 1985.

CARSEL, R.F.; NIXON, W.B.; BALLANTINE, L.G. Comparison of Pesticide Root Zone Model predictions with observed concentrations for the tobacco pesticide metalaxyl in unsaturated zone soils. Environmental Toxicology and Chemistry, Oxford, v.5, p.345-353, 1986.

CLEMENTE, R.S.; PRASHER, S.O.; BARRINGTON, S.F. Pestfade: a new pesticide fate and transport model: model development and verification. 
Transactions of the ASAE, Michigan, v.36, n.2, p.357-367, Mar./Apr. 1993.

CORRÊA, M.M. Mobilidade e retenção do inseticidanematicida sulfona de aldicarbe em colunas de solo de regiões produtoras de batata de Minas Gerais. Lavras : UFLA, 1996. 76p. Dissertação de Mestrado.

DOMAGALSKI, J.L.; DUBROVSKY, N.M. Pesticide residues in ground water of the San Joaquin Valley, California. Journal of Hydrology, Amsterdam, v.130, p.299-338, 1992.

GENUCHTEN, M.Th. van. A closed-form equation for predicting the hydraulic conductivity of unsaturated soils. Soil Science Society of America. Journal, Madison, v.44, n.5, p.892-898, Sept./Oct. 1980.

GORING, C.A.I. Control of nitrification by 2-chloro-6(trichloro-methyl) pyridine. Soil Science, Maryland, v.93, n.3, p.211-218, Mar. 1962.

HUTSON, J.L.; WAGENET, R.J. LEACHM: Leaching Estimation and Chemistry Model. A process-based model of water and solute movement, transformations, plant uptake and chemical reactions in the unsaturated zone. Version 3. Ithaca : Cornell Univ., 1992. Paginação irregular.

JARVIS, N.J. The MACRO model (Version 3.1): technical description and sample simulations. Uppsala : Swedish Univ. of Agricultural Sciences, 1994. 51p. (Reports and Dissertations, 19)

JONES, R.L.; BACK, R.C. Monitoring aldicarb residues in Florida soil and water. Environmental Toxicology and Chemistry, Elmsford, v.3, p.9-20, 1984.

JONES, R.L.; BLACK, G.W.; ESTES, T.L. Comparison of computer predictions with unsaturated zone field data for aldicarbe and aldoxycarb. Environmental Toxicology and Chemistry, Elmsford, v.5, p.10271037, 1986

JONES, R.L.; HORNSBY, A.G.; RAO, P.S.C. Degradation and movement of aldicarb residues in Florida citrus soils. Pesticide Science, Oxford, v.23, p.307-325, 1988.

KLEIN, M. PELMO: Pesticide Leaching Model. Schmallenberg : Fraunhofer Institute, 1995. 103p.

LEONARD, R.A.; KNISEL, W.G.; STILL, D.A. GLEAMS: Groundwater Loading Effects of Agricultural Management Systems. Transactions of the ASAE, Michigan, v.30, n.5, p.1403-1418, Sept./Oct. 1987.

LIGHTFOOT, E.N.; THORNE, P.S.; JONES, R.L.; HANSEN, J.L.; ROMINE, R.R. Laboratory studies on mechanisms for the degradation of aldicarb, aldicarb sulphoxide and aldicarb sulphone. Environmental Toxicology and Chemistry, Elmsford, v.6, p.377-394, 1987.

LIMA, L.A. Polietileno, compactação e tratamento químico na redução das perdas de água por infiltração em canais de irrigação. Pesquisa Agropecuária Brasileira, Brasília, v.22, n.11/12, p.1201-1207, nov./ dez. 1987.

LOAGUE, K.M.; GREEN, R.E. Statistical and graphical methods for evaluating solute transport models: overview and application. Journal of Contaminant Hydrology, Amsterdam, v.7, p.51-73, 1991

MULLINS, J.A.; CARSEL, R.F.; SCARBROUGH, J.E.; IVERY, A.M. PRZM-2, a model for predicting pesticide fate in the crop root and unsaturated soil zones: users manual for release 2.0. Athens : EPA, 1993. Não paginado.

NICHOLLS, P.H.; BROMILOW, R.H.; ADDISCOTT, T.M. Measured and simulated behaviour of fluometuron, aldoxycarb and chloride ion in a fallow structured soil. Pesticide Science, Oxford, v.13, p.475-483, 1982a.

NICHOLLS, P.H.; WALKER, A.; BAKER, R.J. Measurement and simulation of the degradation of atrazine and metribuzin in a fallow soil. Pesticide Science, Oxford, v.12, p.484-494, 1982b.

NOFZIGER, D.L.; HORNSBY, A.G. A micro-computer based management tool for chemical movement in soil. Applied Agricultural Research, New York, v.1, p.50-56, 1986.

OLIVEIRA, J.B. de; JACOMINE, P.K.T.; CAMARGO, M.N. Classes de solo. In: OLIVEIRA, J.B. de; JACOMINE, P.K.T.; CAMARGO, M.N. Classes gerais de solos do Brasil. Jaboticabal : FUNEP, 1992. p. $95-190$.

PARRISH, R.S.; SMITH, C.N.; FONG, F.K. Tests of the Pesticide Root Zone Model and the aggregate model for transport and transformation of aldicarb, metolachlor, and bromide. Journal of Environmental Quality, Madison, v.21, p.685-697, 1992. 
PENNELL, K.D.; HORNSBY, A.G.; JESSUP, R.E.; RAO, P.S.C. Evaluation of five simulation models for predicting aldicarb and bromide behavior under field condictions. Water Resources Research, Washington, DC, v.26, n.11, p.2679-2693, Nov. 1990.

PIFFER, R.; RIGITANO, R.L.O. Lixiviação e degradação do inseticida aldicarbe em dois diferentes solos. Ciência e Prática, Lavras, v.15, n.4, p.355-363, out./dez. 1991.

RAO, P.S.C.; JESSUP, R.E. Sorption and movement of pesticides and other toxic organic substances in soils. In: NELSON, D.W.; ELRICK, D.E.; TANJI, K.K. (Eds.). Chemical mobility and reactivity in soil systems. Madison : SSSA, 1983. p.183-201.

SHIRMOHAMMADI, A.; KNISEL, W.G. Evaluation of the GLEAMS model for pesticide leaching in Sweden.
Journal of Environmental Science and Health, New York, v.29-A, n.6, p.1167-1182, 1994.

WALKER, A.; HOLLIS, J.M. Prediction of pesticide mobility in soils and their potential to contaminate surface and groundwater. In: HEWITT, H.G.; CASELEY, J.; COPPING, L.G. (Eds.). Comparing glasshouse and field pesticide perfomance. Canterbury : BCPC, 1994. p.211-224. (Monograph, 59).

WALKER, A.; WELCH, S.J.; MELACINI, A.; MOON, Y.H. Evaluation of three pesticide leaching models with experimental data for alachlor, atrazine and metribuzin. Weed Research, Oxford, v.36, n.1, p.37-47, Feb. 1996.

WORTHING, C.R. The pesticide manual: a world compendium. Croydon : BCPC, 1983. 695p. 